\title{
Intelligent Decisions Modeling for Energy Saving in Lifts: An Application for Kleemann Hellas Elevators
}

\author{
Vasilios Zarikas ${ }^{1}$, Nick Papanikolaou ${ }^{2}$, Michalis Loupis ${ }^{3}$, Nick Spyropoulos ${ }^{4}$ \\ ${ }^{1}$ Department of Electrical Engineering, Theory Division, Academic Institute of Technology of Lamia, ATEI Lamias, Lamia, Greece \\ ${ }^{2}$ Department of Electrical Engineering, Power Electronics Division, ATEI Lamias, Lamia, Greece \\ ${ }^{3}$ Department of Electrical Engineering, Informatics Division, ATEI Lamias, Lamia, Greece \\ ${ }^{4}$ Research and Development Division, Kleeman Hellas, Kilkis, Greece \\ Email: vzarikas@teilam.gr
}

Received February 24, 2013; revised March 26, 2013; accepted April 9, 2013

Copyright (c) 2013 Vasilios Zarikas et al. This is an open access article distributed under the Creative Commons Attribution License, which permits unrestricted use, distribution, and reproduction in any medium, provided the original work is properly cited.

\begin{abstract}
The present work proposes a methodological approach for modeling decisions regarding energy reduction in an elevator. This is achieved with the integration of existing as well as acquired knowledge, in a decision module implemented in the electronics of an elevator. So far, elevators do not exploit information regarding their recent usage. In the developed system decisions are driven based on information arising from monitoring the use of the elevator. Monitoring provides various records of usage which consequently are used to predict elevator's future usage and to adapt accordingly its functioning. Till now, there are only elevators that encompass in their electronics algorithms with if then rules in order to control elevator's functioning. However, these if then rules are based only on good practice knowledge of similar elevators installed in similar buildings. Even this knowledge which unavoidably is associated with uncertainty is not encoded in a mathematically consisted way in the algorithms. The design, the implementation and a first pilot evaluation study of an elevator's intelligent decision module are presented. The study concludes that the presented application sufficiently reduces energy consumption through properly controlled functioning.
\end{abstract}

Keywords: Elevators; Energy Consumption Reduction; Energy Engineering; Applied Bayesian Networks; Applied Decision Networks; Applied Influence Diagrams; Applied Intelligent Decisions; Fuzzy Rules

\section{Introduction}

A large number of techniques in the field of artificial intelligence used to represent knowledge and/or describe decision problems: production rules, semantic nets, Bayesian networks, frameworks, scripts, statements, logic, fuzzy logic and possibility theory, causal networks, among others. They have been shown to be effective especially in domain dependent decision tasks. The choice of a particular technique is based on two main factors: the nature of the application and the skills of the user/designer.

The present work describes a specific technological application of bayesian networks. The bayesian networks that were implemented, incorporated certain and uncertain knowledge as well as fuzzy rules in order to guide the proper functioning of an elevator with respect to energy consumption minimization. bayesian networks are well-known and efficient mathematical models based on subjective probability theory to model knowledge and making inference. Moreover, probabilistic modeling among these observed parameters of interest are central to science. In order to algorithmically consider causal probabilistic relations, the relations must be placed into a representation that supports manipulation.

Fuzzy rules were used because fuzzy logic is very close to the expert's reasoning. Its utilization became very popular in attempting to resolve the problems of imprecision and uncertainty. It is easier for engineers to express their knowledge using fuzzy If-Then rules instead of using conditional probabilities. Thus, experts can express their knowledge through a number of fuzzy rules to describe the observations and their impact into the problem.

A common problematic feature of these bayesian models is that a very detailed amount of information is used to fill all the involved conditional probability tables (CPTs). Our approach suggests a simple working solution for modeling this particular decision problem of energy saving. This approach determines the CPTs taking 
probabilities from the measured frequencies of the last week usage of the elevator. In addition the proposed solution suggests a particular novel way for assigning fuzzy linguistic values, extracted from rules, in order to fill conditional probability tables in these BBNs.

Based on searches of the relevant literature, no previous work was found suggesting a bayesian decision system for driving the functioning of an elevator. However, there are some interesting applications of intelligent control units in elevators [1-6].

The paper is organized into the following sections: the second section presents a description on Bayesian networks influence diagrams and Fuzzy rules. The third section provides a description of the designed and developed decision networks. The fourth section describes the first evaluation of the system. Finally, the fifth section discusses the results of the developed tool, outlining is also the main conclusions of the study.

\section{Bayesian Networks and Fuzzy Logic}

The imprecise and insufficient information that always appears in technology cannot be incorporated in models using the framework of "if-then" rules. However human experts are able to reach decisions with a high level of validity even if the input data are almost always uncertain. This fact led to the development of mathematical models capable to manipulate pieces of information that are associated with an uncertainty value, named Bayesian networks.

Early developed rule based systems were consisted of a knowledge base and an inference system. The knowledge base is a set of rules of the form "If A (with certainty 1) then B (with certainty 1)”. Implementing an inference system it is possible to combine these rules and other observations in order to finally reach a decision. Although this framework can tackle a considerable amount of adaptation functioning it cannot be generalized in order to include cases where the condition (A) or/and the act (B) is subject to an uncertainty level.

The construction of a consistent mathematical framework that is allowed to incorporate uncertain pieces of information into a plan of reasoning exists and is the so called belief network or Bayesian network or causal graph [7-11]. It is a graphical representation of a problem domain, which consists of informational nodes (pieces of information) that are known with certainty or with an uncertainty described by a subjective probability. Subjective probabilities express measures of a person's belief, given a certain knowledge background carried by that person. This notion of probability differs from the most used classical probability. The subjective or Bayesian probabilities can describe a value of belief to unique events that are not repeatable. Thus, a subjective prob- ability $P(O \mid X, C)$ describes the subjective estimate (belief) of certainty of an event $O$, given as known that the event $X$ occurred and given a certain background knowledge $C$.

An important property of this framework is the fact that the direction of probabilistic inference can be reversed. This is allowed due to the Bayes' theorem. Suppose that we know the belief of the influence of a hypothesis $H$ on the observable evidence $E, P(E \mid H, C)$. Then Bayes' theorem allows us to compute the belief on the influence of $E$ on $H$ (the so called posterior probability) as a function of the prior probability $P(H \mid C)$.

$$
P(H \mid E, C)=\frac{P(E \mid H, C) P(H \mid C)}{P(E \mid C)}
$$

It is now possible, through this theorem, for domain experts to provide estimates of subjective probabilities in the causal direction and calculates beliefs in the backward the so called diagnostic direction i.e. learning the belief in a hypothesis given the relevant evidences. In real life problems we face situations where there are relationships among a large number of variables. A Bayesian network is a representation of such cases.

Formally, a Bayesian network is a graph with the following elements and properties 1) A set of variables (shown as nodes in the diagram). Each variable has a set of mutually exclusive states; 2) A set of directed edges between these variables; 3 ) The variables and the edges form a directed acyclic graph and 4) To each node-variable there is attached a conditional probability that depends on the parents of the node.

Thus, the design of a Bayesian network is required to draw arcs from cause variables to their immediate effects. In this way causal relationships reveal the conditional dependencies and independencies. After constructing the network based on our prior knowledge and data, appropriate algorithms [7,12-14] exist, in order to determine various probabilities from the network. Probabilistic inferences can be produced with the estimation of a certain probability of interest from our model.

An additional issue is the combination of Utility theory and Bayesian graph theory. This synthesis formulates Decision theory [15-18]. Utility theory provides the axiomatic framework for consistency among preferences and decisions. The axioms introduce the concept of a lottery which is an uncertain situation with various outcomes assigned with a probability of occurrence. Then a set of rules define which outcome is preferred from which lottery. Accepting these axioms we can always define a utility function. A utility function is a scalar that assigns a cardinal scale to each outcome and decision indicating its desirability. The preferred set of decisions is the one that maximizes the expected utility given, with 
known uncertainty, the various relevant parameters.

For a decision problem where there is a set of mutually exclusive decision states $D_{i}$ with $i=1, \cdots, n$ and one determining variable $V$ with possible states $V_{j}$ with $j=1, \cdots, m$ (an hypothesis that drives the decision). Further, we are interested in cases with non intervening decision states or in other words decisions that their states does not have any correlation with $P(H)$. The determining variable $V$ is part of Bayesian network, and has various parents and child nodes in general.

Constructing an influence network [7] or otherwise called decision network (a Bayesian network with utilities and decision/action nodes) means finally to set the values of a utility table that determine for each action $D_{i}$ and each state $V_{j}$ a number that expresses the utility $U\left(D_{i}, V_{i}\right)$ that we gain. Then the expected utility for taking actions is

$$
E U\left(D_{i}\right)=\sum_{j} U\left(D_{j}, V_{j}\right) P\left(V_{j}\right)
$$

The preferred decision is associated with the action that gives the maximal expected utility $M(D)$.

$$
M(D)=\max _{i} E U\left(D_{i}\right)
$$

For cases, we have $N$ decision/action variables $D^{(k)}$ with determining variables $V^{(k)}$ where $k=1, \cdots, N$, and we would like to drive a decision based on all the combinations of actions, then it is straightforward to generalize for a new expected utility as follows

$$
\begin{aligned}
& E U\left(D^{(1)}, D^{(2)}, \cdots, D^{(N)}\right) \\
& =\sum_{k} w_{(k)} \sum_{V_{j}^{(k)}} U_{k}\left(D^{(k)}, V^{(k)}\right) P\left(V^{(k)}\right)
\end{aligned}
$$

where $w_{(k)}$ are the weights that reflect the significance of its action variable with respect to its other. It is important to note that we have treated the various combinations of action states $D_{i}^{(1)}, D_{j}^{(2)}, \cdots, D_{l}^{(N)} \in D^{(1)} \times D^{(2)} \times \cdots \times D^{(N)}$ belonging to different action variables as states of one action variable which is the Cartesian product of the $N$ decision/action variables.

Here we assume that in the previous decision modeling the actions have no impact on the variables in the networks which affect the belief on the hypothesis nodes. In other words we described the framework for sets of non-intervening actions.

Finally, the various nodes in an influence diagram, can be deterministic informational nodes, statistical results or beliefs given as probability distributions, utilities and action (or otherwise called decision) nodes. Decision nodes represent possible actions, informational nodes represent pieces of certain or uncertain relevant knowledge while utility nodes encapsulate designer's preferences, goals etc. Such a diagram then represents a deci- sion basis.

For the problem of making technological decisions, engineers are usually thinking in order to reach a decision or suggest a solution in a form of if-then rule, i.e. Furthermore, a rule-base is more transparent and understandable for engineers. Fuzzy logic is based on fuzzy if-then rules which have the general form "IF $X$ is $A$ THEN Y is B," where A and B are fuzzy sets. A fuzzy set is a set containing elements that have varying degrees of membership in the set. Elements in a fuzzy set can also be members of other fuzzy set on the same universe $[19,20]$, because their membership need not be complete.

Following a knowledge representation viewpoint, a fuzzy if-then rule is a scheme for capturing knowledge that involves imprecision. A key property of reasoning using these rules is its partial matching capability, which enables an inference to be made from a fuzzy rule even when the rule's condition is only partially satisfied.

Randomness describes the uncertainty in the occurrence of an event while fuzziness describes the ambiguity of an event. In classical sets there is no uncertainty, hence they have crisp boundaries, but in the case of a fuzzy set, since uncertainty occurs, the boundaries may be ambiguously specified. The membership function for a set maps each element of the set to a membership value between 0 and 1 and uniquely describes that set. The values 0 and 1 describe "not belonging to" and "belonging to" a conventional set respectively; values in between represent "fuzziness." The determination of the membership function is subjective to varying degrees depending on the situation. It is determined on an individual's perception of the data in question and does not depend on randomness. The latter is a significant point and distinguishes fuzzy set theory from probability theory. The membership functions that constitute the fuzzy sets which describe the inference of the fuzzy rules are depicted in Equation (5). A numerical value of each fuzzy set is produced after defuzzification with the Center of Area method. The produced numerical value is used to fill the probabilities in CPTs.

\section{Designing the Bayesian Network}

Some common critics about applied Bayesian networks concern the necessity of filling correctly a lot of conditional probability tables. On the other hand, the appearance of all these probability tables make this decision tool extremely precise, expressive and mathematically consisted. It makes also profound emphatically to the decision builder and/or the interviewed expert how many pieces of information are involved for precise decision making. This set of probabilities by no means can be disregarded unwisely for the sake of simplicity or a fault decision will be driven. On the other hand, experts com- 
plained that the human brain does not work in this way and even scientists (not experienced in "Bayesian language") cannot easily report safely all these numbers in order to describe a domain knowledge. Thus, it is vital for the construction of a proper decision network, a correct set of all involved probabilities. This is a task that should be carried from the designer of the bayesian network who usually is a person familiar with the bayesian mathematical framework. Engineers should be allowed to report their knowledge in the form of fuzzy if then rules, thus avoiding misunderstandings with the bayesian reasoning. In the present work engineers reported relevant knowledge in the form of if then rules. A practical solution of this problem is presented in this work for the decision model in study.

The decisions that our decision module has to take are two. First if the elevator after its last use will be on a full activation mode (FM), in a standby mode (SM) or in an off mode (OM). Second the engine should decide in which of the n-th floors the elevator cabinet should rest after its last usage. Both these decisions will be driven according the recorded knowledge of the last week's usage. More specifically both these decision are affected by the following informational deterministic and decision nodes.

- Date.

- Twenty four times seven times $n(24 \times 7 \times n)$ informational nodes that provide the frequency of calls (number of calls of ith floor over total calls of all $n$ floors at the same timezone) from every one of the n-th floors, each for every time zone of each one of the seven days.

- Node expressing the reasoning of common practice fuzzy rule that refers to the preference of the floor chosen to rest, for either a specific season or day or timezone (this helps to make better prediction in cases such as the end of the two weeks Christmas vacation period and the beginning of a full working week).

- Subjective probability expressing the preference of the medium floors as far as the energy consumption is concerned.

- Twenty four times seven $(24 \times 7)$ informational nodes that provide the normalized duration of idle use (idle time length over the time duration of the time zone = 1 hour) each for every time zone of each one of the seven days.

- Node expressing the reasoning of common practice fuzzy rule that refers to the preference of the type of functioning mode (FM or SM or OM) for either a specific season or day or timezone (this helps to make better prediction in cases such as the end of the two weeks Christmas vacation period and the beginning of a full working week).

- Subjective probability expressing the preference of the FM status compared to SM and SM compared to OM mode as far as the user annoyance is concerned (this information makes a difference in case of almost equal balance among two modes).

- One utility comprising the overall utility driven by historic data. Utility values are associated on each of the three states the elevator will have to choose: a full activation mode (FM), in a standby mode (SM) or in an off mode (OM).

- One utility comprising the utility driven by the preference of each of the three states the elevator: a full activation mode (FM), in a standby mode (SM) or in an off mode (OM).

- One central multi attribute utility comprising the overall utility of each of the three states the elevator: a full activation mode (FM), in a standby mode (SM) or in an off mode (OM). This utility is driven be the positive effect of the historic data utility and the negative effect of the preference utility.

- Decision node concerning which of the three states the elevator will choose to rest: a full activation mode (FM), in a standby mode (SM) or in an off mode (OM).

Note that in the implemented system we have defined 24 timezones per day and seven days per week. The history records concern last 7 days period data.

In Figure 1, a small simplified part (it is not possible to present the full bayesian network due to its size) of the real constructed bayesian network is presented. Note that the preference utility expresses the fact that the FM state is preferable for users contrary to the suggestion of the historic data that choose the most economic mode as far as energy consumption is concerned. The multiattribute utility realizes the trade off of the two utilities according to the designer's tuning.

The fuzzy rules that are integrated into the bayesian network are of the general form:

"If the season is A and the day is B and the time zone is $\mathrm{C}$ then the floor $\mathrm{N}$ (much less, less, more, much more) preferable”.

"If the season is A and the day is B and the time zone is $\mathrm{C}$ then the mode (FM or SM or OM) is (much less, less, equally, more, much more) preferable”.

In order to translate these fuzzy rules into probabilities through the defuzzification process we have to define first the membership function in use.

$$
\mu\left(B_{n}\right)=\left\{\begin{array}{l}
\frac{x}{x_{n}-x_{n-1}}+\frac{x_{n-1}}{x_{n-1}-x_{n}}, x_{n-1} \leq x \leq x_{n} \\
\frac{x}{x_{n}-x_{n+1}}+\frac{x_{n+1}}{x_{n+1}-x_{n}}, x_{n} \leq x \leq x_{n+1}
\end{array}\right.
$$

where $n=1,2, \cdots, 5$ with $x_{n}=n / 6$. In Equation (5), $x$ takes values from 0 to 1 and B1 = v.v.weak, B2 = v.weak, 


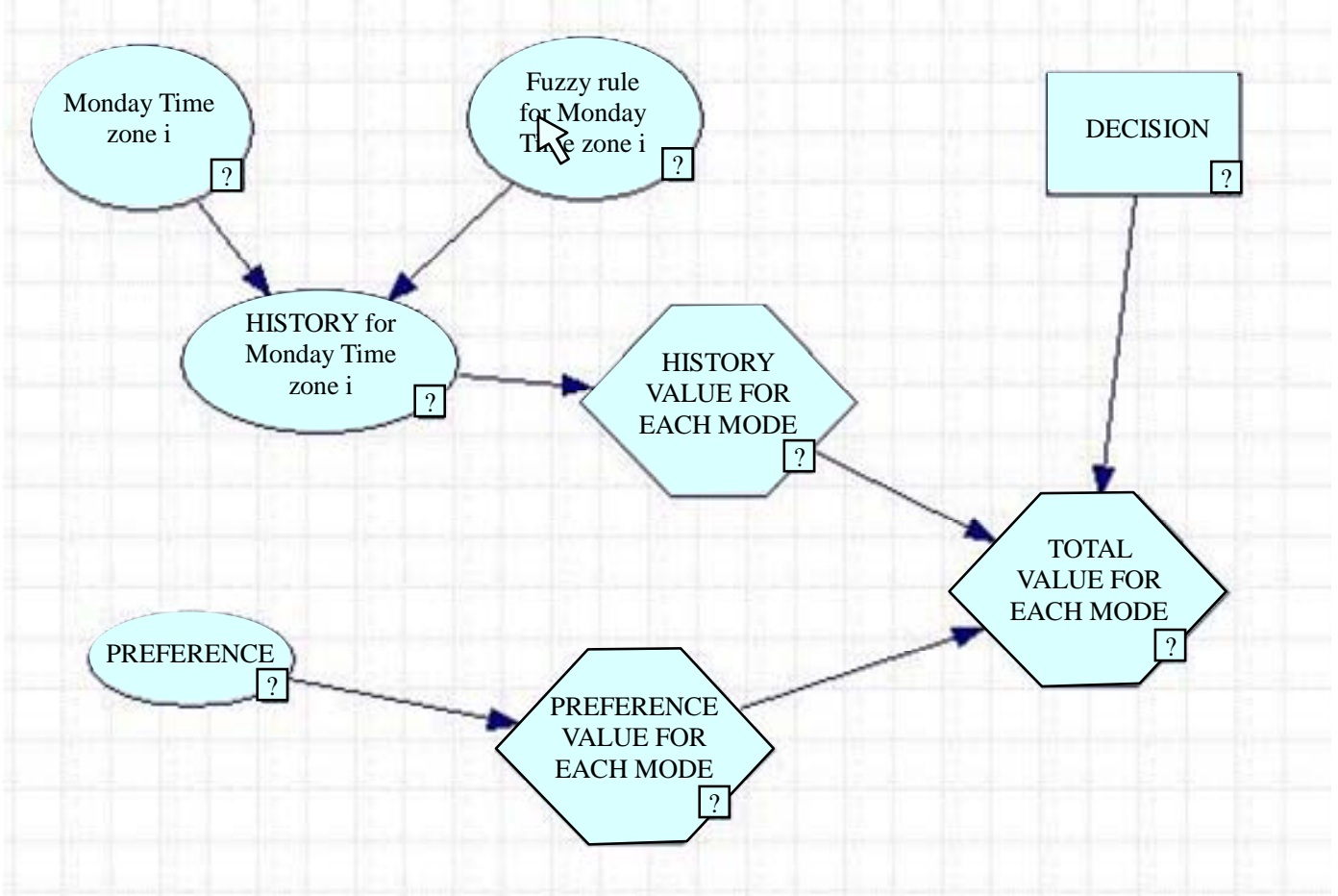

Figure 1. Simplified influence diagram for the decision concerning functioning mode.

B3 = weak, B4 = weak-med, B5 = medium, B6 = med-high, B7 = high, B8 = v.high, B9 = v.v.high. Central values are given in Equation (6).

$$
\text { Central }\left[\mu\left(B_{n}\right)\right]=\left\{\begin{array}{l}
0, \quad- \\
0.15, B_{1}=\text { much less } \\
0.35, B_{2}=\text { less } \\
0.5, B_{3}=\text { equal } \\
0.65, B_{4}=\text { more } \\
0.85, B_{5}=\text { much more } \\
1, \quad-
\end{array}\right.
$$

Based now, on the defined membership functions, linguistic values contained in the rules are transferred to numerical values in order to fill the conditional probability tables, through the defuzzification approach of fuzzy logic. In order to show how the probability tables for BBNs are developed using the above type of if-then rules, a generic approach is provided. Let's consider the following rule for the assessment of preferability to $S M$ mode during a timezone: "If the day is $X$ and the time zone is $Y$ then the mode (SM) is (more) preferable". This rule suggests information capable to provide probabilities for the conditional probability table $(C P T)$ between the preferability of mode $S M$ and the observables $X, Y$.

$$
P(S M \mid X, Y)=\left\{\begin{array}{l}
\mu\left(B_{4}\right), \text { timezone }=Y ; \\
1 / 3, \quad \text { timezone } \neq Y .
\end{array}\right.
$$

Also

$P(\operatorname{mode} \mid X, Y)=$

$\left\{\begin{array}{l}\frac{1-\mu\left(B_{4}\right)}{2}, \text { timzone }=Y, \text { mode }=O M ; \\ \frac{1-\mu\left(B_{4}\right)}{2}, \text { timzone }=Y, \text { mode }=F M ; \\ 1 / 3, \quad \text { timzone } \neq Y, \text { mode }=F M \text { or mode }=O M .\end{array}\right.$

These probabilities Equations (7) and (8) concern only day $\mathrm{X}$ in the simple case that there are no other rules for this day.

Since all informational nodes affect the final decision naturally a first approach is to connect all informational nodes to a central node representing a total utility. However, this leads to a huge and quite confusing probability table. For example, in a case of entering values for a new rule, it would be necessary to change the values for the already entered rules. Furthermore, a table containing values gained from more than two or three nodes is highly inflexible. It's not possible to distinguish the values between the different nodes. Therefore intermediate deterministic nodes are necessary for a solvable network topology, see Figure 1.

\section{Evaluation}

Genie tool has provided the C code which consequently 
has been integrated for research purposes in the electronics of the control unit see Figure 2.

\subsection{The VDI Directive}

The reduction of energy consumption in modern lifts has become a critical parameter for their commercial competitiveness, especially in the EU market. For this reason, all important lift developing companies are making Research and Development efforts through the introduction of improved driving techniques (in terms of the electromechanical part) as well as of "smart" energy management methods (in cases of multiple installed lifts at the same building).

However, the determination of the exact energy consumption for a lift is a rather complicated issue, taking into account their stochastic use and the variety of the load and of the total trip-even on an annual basis. Hence, for the ensuring of the healthy competitiveness in the lift market specific and common energy consumption measuring rules have been set. To this direction VDI 4707 is a commonly accepted guideline [21] which describes a transparent method for the assessment and classification of the power requirement and consumption of lifts. According to VDI 4707 the lift consumption can be distinguished to the following categories: A. Travel Demand, is the total energy consumption of the lift during trips at specified trip cycles with a defined load. Travel demand is expressed as energy consumption per traveled distance multiplied by load weight, $\mathrm{Wh} /(\mathrm{m} \cdot \mathrm{kg})$. B. Standby Demand, is the total energy consumption of the lift in standby mode. Relevant studies in this issue [22-25], have highlighted that the standby mode consumption is a very important part of the annual energy consumption, which may reach (in some cases of rarely used lifts) even the 80 percent. Standby demand is expressed as power consumption, (W).

Today commercial lifts are adopting energy saving methods in order to be included in the higher possible energy category (according to VDI 4707). These energy saving techniques are aiming to the reduction of energy consumption in terms of travel demand as well as of standby demand. In more details, travel demand is reduced by using regenerative breaking through AC/DC/ AC power electronic drives as Figure 3. Presents [26,27]. For the standby mode energy saving is achieved by switching off lighting and inverter electronic circuits. Additionally, some modern lifts use more sophisticated software in order to cut down standby consumption; these lifts are programmed in such a way, so as to power off during intervals that are not used. The main difficulty in this type of lift control is that after powering off a critical time interval is necessary until the lift comes again in full operation (reset of electronic circuits, self test, etc). Moreover, this time interval protects the inverter from frequent restarts which would jeopardize its health status (mainly due to the start up overcurrents). Nevertheless, this sophisticated operation is not adaptive, but it is standard for each building category. In the present work the option of implementing an adaptive controller for lifts is investigated. The proposed control algorithm modifies the standby and power off modes time intervals according to the lift use-as daily as seasonalachieving so lower annual energy consumption. Furthermore, the proposed algorithm aims also at the travel

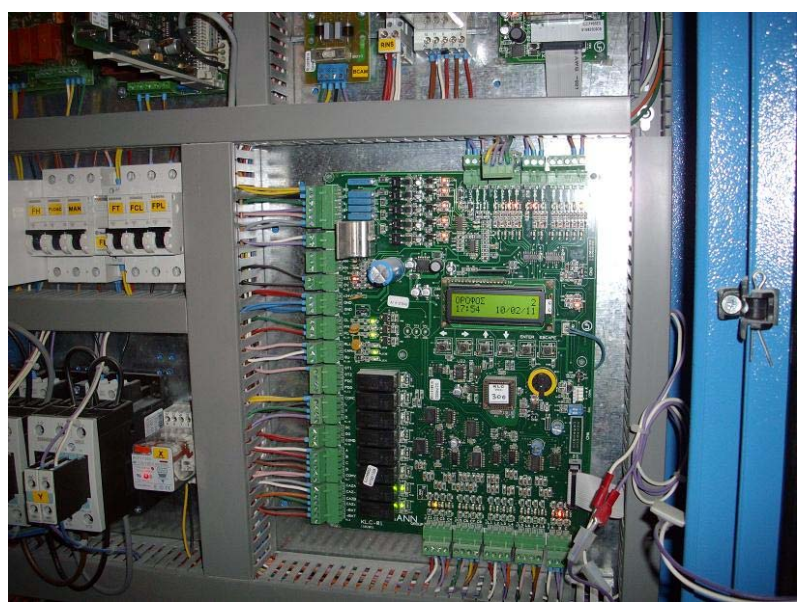

Figure 2. Control unit.

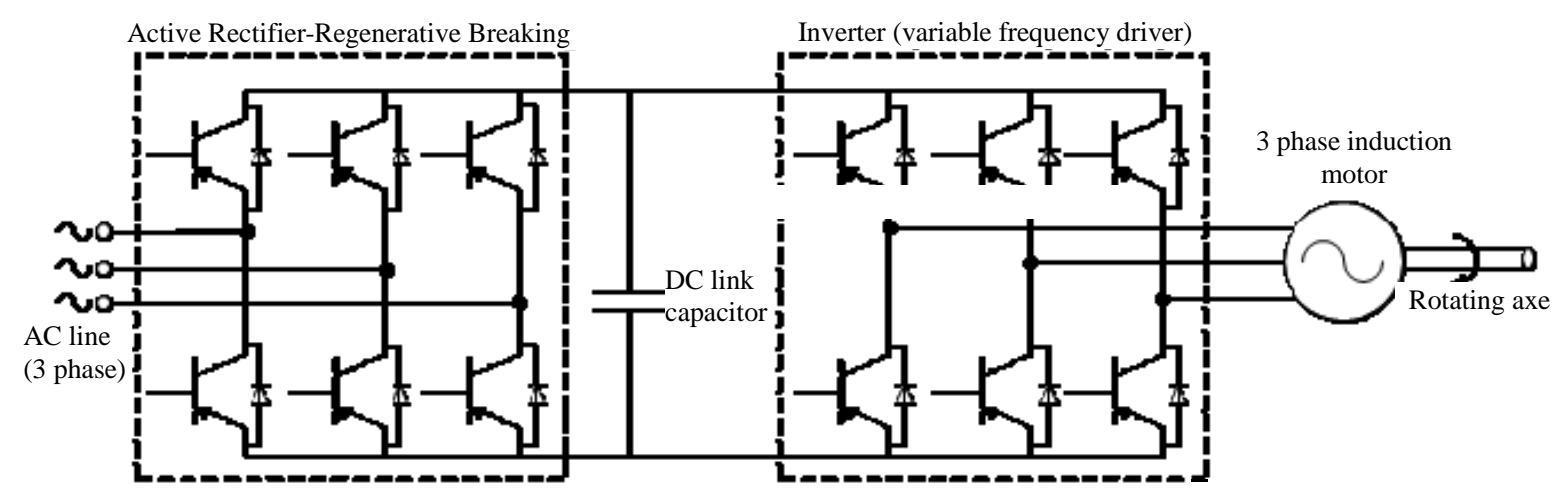

Figure 3. AC/DC/AC inverter drive schematic with regenerative breaking. 
demand reduction through the decrease of the annual lift traveled distance.

The proposed algorithm has been tested to the following elevator type-derived by VDI 4707 sample calculation:

- Type of building: Residential block/ doctor's practice.

- Nominal load: $320 \mathrm{~kg}$.

- Speed: $0.63 \mathrm{~m} / \mathrm{s}$.

- Stops: 5.

- Vertical rise: $11.2 \mathrm{~m}$.

- Trips per day: approximately 100.

- Travel distance: 1,134 m per day.

- Travel demand: $8.93 \mathrm{mWh} /(\mathrm{m} \cdot \mathrm{kg})$.

- Standby demand: $200 \mathrm{~W}$.

- Usage category (according to VDI 4707): category 1.

- Travel energy demand per day: $8.93 \mathrm{mWh} /(\mathrm{m} \cdot \mathrm{kg})$ $1134 \mathrm{~m} 320 \mathrm{~kg}=3.24 \mathrm{kWh}$.

- Standby energy demand per day: $110 \mathrm{~W} 23 \mathrm{~h}=2.59$ kWh.

- Total energy demand per day: $3.24 \mathrm{kWh}+2.59 \mathrm{kWh}$ $=5.82 \mathrm{kWh}$.

- - Specific energy demand: 5.82 kWh/(1134 m 320 kg) $=16.05 \mathrm{mWh} /(\mathrm{m} \cdot \mathrm{kg})$.

- Energy efficiency class (according to VDI $4707 \mathrm{cal}-$ culation): class F.

\subsection{Test Procedure/Results}

Kleemann Hellas was founded in 1983, based on the know-how and licence of the german company Kleemann HUBTECHNIK GmbH. The head office of the company is based in the Industrial Area of Kilkis in Northern Greece. Kleemann company's activities concern both the manufacturing and trading of Complete Lift Systems. Kleemann is enlisted among the largest companies of the lift industry in the European and international market (more than 12,000 new systems or three percent of the world's new lift units annually).

Tests were performed for a Kleeman elevator carrying the developed research decision support system on its electronic control unit. The evaluation took place in the Tower for Experiments based on the headquarters of the company. This tower belongs to the main industrial campus in Kilkis city, Macedonia, Greece, see Figure 4. The test procedure comprised two main scenarios: 1) A heavy load scenario (meaning 100 trips/day) with two peaks morning (08:00 - 10:00) and afternoon (16:00 18:00) and 2) An average use scenario (meaning 50 trips/day and less dense peaks). Each scenario was followed for one week. The final outcome of this pilot study evaluation was: A fourteen percent energy saving, for the heavy load scenario, was achieved compared to the energy consumption of the same elevator with a conventional control unit. In addition, a five percent energy saving, for

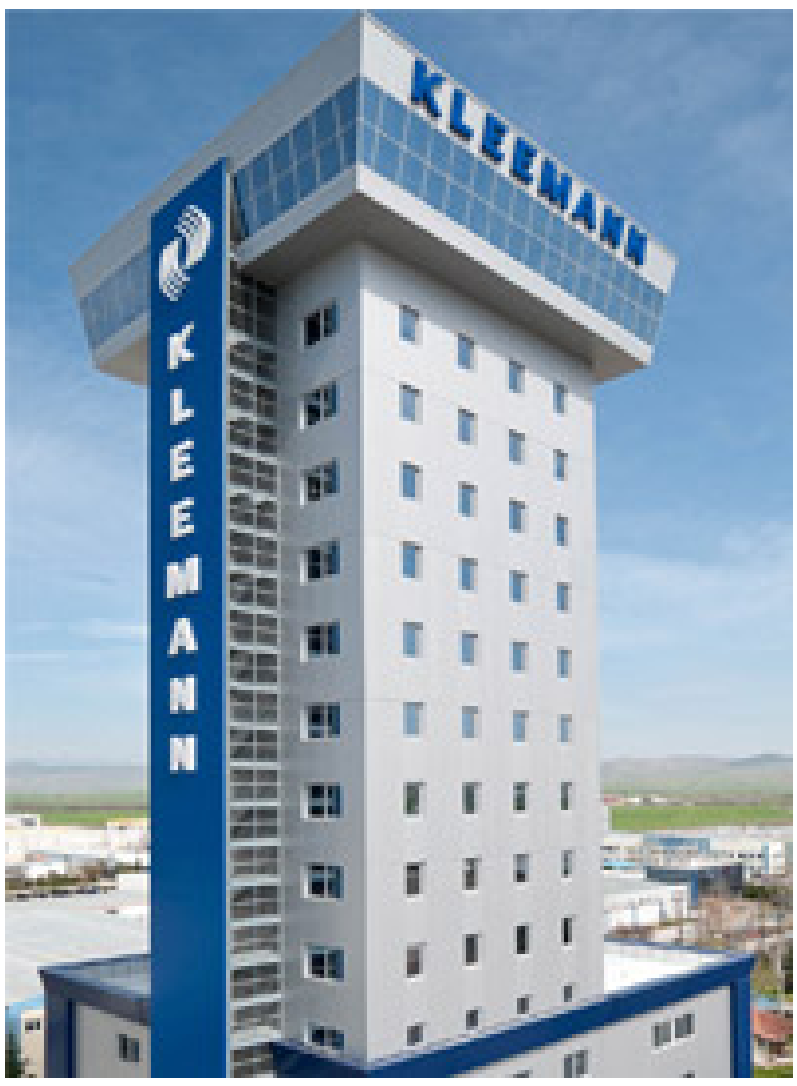

Figure 4. Kleemann tower for elevators testing.

the average load scenario, was achieved compared to the energy consumption of the same elevator with a conventional control unit.

These first results together with an analytic list of all decisions that have been performed by the decision system will be the input for improving the whole system during the second evaluation run.

\section{Discussion}

Engineers as experts have reported certain and uncertain scientific knowledge in the form of fuzzy rules. The decision system suggests actions based on their reported fuzzy rules and the frequencies that the control unit evaluates from the last week usage of the elevator. The decision support system encapsulates two specific topology bayesian networks which were designed for the two main decisions that ensure energy saving.

After construction of Bayesian network using the Genie tool, a number of test cases have been examined in order to set evidences to the network and illustrate its decision making capabilities. Specifically, one hundred fifty (150) trips each associated with the relevant decision making actions were performed. The decision making capabilities of the system were tested and they will guide the reforming of the whole system in order to 
eliminate false responses. However even in this first pilot study a non negligible energy saving was achieved.

The developed system gives a front-end decision system about the functioning of elevators with the aim to save energy. Of course, more trials and real tests are needed for a large number of cases in order to confirm or improve the system. Future work will be directed towards this direction. The aim of this research paper was two fold. First, a new techniques for integrating into BNs fuzzy if-then rules was proposed and second an efficient modeling and reasoning concerning the integration of all informational nodes into the network with a specific workable and solvable topology was presented.

This approach for modeling decisions has several advantages: 1) a graphical way of encoding the information is used; 2) certain and uncertain pieces of knowledge can be consistently incorporated and interweaved; 3) nodes in these diagrams are able to represent information coming from engineers/experts and historic data; 4) the system can be adjusted to different decision policies and strategies; 5) beliefs associated with some of the informational nodes can be altered dynamically and a new decision based on the new evidences can be driven; 6) decisions are not hard-coded into the system, this means that the influence diagram comprises a high level description of the decision reasoning that could be easily modified, customized and re-used; and, 7) if during the intermediate evaluation of the application, new knowledge (rules) has to be built in, the designer either simply corrects the various conditional probabilities or more alters the topology of the network and the probabilities. Some difficulties and disadvantages are: 1 ) the designer or developer needs to understand the Bayesian reasoning; 2) the designer or developer needs to test the network for sensitivity in order to check if the changes of the various probabilities have the correct and desired impact on the utilities driven decision. However, the latter is not a difficult task since for most of the cases only a small part of the network is activated.

Novel ideas that have been materialized in the present work are: 1) Experts/engineers have not been involved for the probability assignments but only for reporting fuzzy rules; 2) Fuzzy rules have been translated to probabilities according to a specific technique; 3) Historic data concerning the last week usage of elevator alter non trivially the functioning of the elevator; 4) There is an intermediate layer of utilities that transfer their values to a central utility node

A particular set of cases were studied as a pilot preliminary study. In upcoming work, more tests and trials have to be made for model validation. Future work will be focused to analyze and implement this approach in other type of elevators too as well as on a different context, i.e. large buildings with more than one elevator.

\section{Acknowledgements}

This work has been supported by the research program "Less energy consumption in elevators (LESS)", Project Code 09SYN-32-829, within the Greek Research Activity "COOPERATION". This is co-financed by the European Union (European Social Fund) and Greek national funds.

\section{REFERENCES}

[1] L. Cui, “An Elevator Intelligent Scheduling Method Using Neural Network Control," International Journal of Digital Content Technology and Its Applications, Vol. 7, No. 3, 2013, pp. 174-181.

[2] T. Chen, Y.-Y. Hsu and Y.-J. Huang, “Optimizing the Intelligent Elevator Group Control System by Using Genetic Algorithm,” Advanced Science Letters, Vol. 9, No. 1, 2012, pp. 957-962. doi:10.1166/asl.2012.2654

[3] P. E. Utgoff and M. E. Connell, "Real-Time Combinatorial Optimization for Elevator Group Dispatching,” IEEE Transactions on Systems, Man, and Cybernetics Part A: Systems and Humans, Vol. 42, No. 1, 2012, pp. 130-146.

[4] N. A. Rahim, H. W. Ping and J. Jamaludin, "A Novel Self-Tuning Scheme for Fuzzy Logic Elevator Group Controller," IEICE Electronics Express, Vol. 7, No. 13, 2010, pp. 892-898.

[5] Y. Cheng, X. Wang and Y. Zhang, “A Bayesian Reinforcement Learning Algorithm Based on Abstract States for Elevator Group Scheduling Systems," Chinese Journal of Electronics, Vol. 19, No. 3, 2010, pp. 394-398.

[6] X.-C. Wang and D.-M. Yang, "Intelligent Algorithm of Elevator Group Control by Statistic Approximation,” Xitong Fangzhen Xuebao/Journal of System Simulation, Vol. 13, 2001, pp. 100-101.

[7] F. V. Jensen, “An Introduction to Bayesian Networks,” UCL Press Limited, London, 2000.

[8] J. Pearl, "Probabilistic Reasoning in Intelligent Systems: Networks of Plausible Inference," Morgan Kaufmann, San Mateo, 1988.

[9] J. Stutz and P. Cheeseman, "A Short Exposition on Bayesian Inference and Probability,” National Aeronautic and Space Administration Ames Research Centre: Computational Sciences Division, Data Learning Group, 1994.

[10] N. Friedman and M. Goldszmidt, "Learning Bayesian Network from Data,” SRI International, Menlo Park, 1998.

[11] D. Heckerman and D. Geiger, "Learning Bayesian Networks,” Microsoft Research, Redmond, 1994, p. 3.

[12] J. Pearl, "Fusion, Propagation and Structuring in Belief Networks,” Artificial Intelligence, Vol. 29, No. 3, 1986, pp. 241-288. doi:10.1016/0004-3702(86)90072-X

[13] J. Pearl, "Evidential Reasoning Using Stochastic Simulation of Causal Models," Artificial Intelligence, Vol. 32, No. 2, 1987, pp. 245-258. doi:10.1016/0004-3702(87)90012-9

[14] J. Pearl and T. Verma, "The Logic of Representing De- 
pendencies by Directed Graphs," Proceedings, AAAI Conference, Seattle, 13-17 July 1987, pp. 374-379.

[15] R. L. Winkler, "An Introduction to Bayesian Inference and Decision," Holt, Rinehart and Winston, Toronto, 1972.

[16] E. J. Horvitz, J. S. Breese and M. Henrion, "Decision Theory in Expert Systems and Artificial Intelligence," International Journal of Approximate Reasoning, Vol. 2, No. 3, 1988, pp. 247-302.

[17] B. W. Morgan, “An Introduction to Bayesian Statistical Decision Processes,” Prentice-Hall Inc., Englewood Cliffs, 1968, p. 15.

[18] J. Pearl, "Influence Diagrams-Historical and Personal Perspectives,” Decision Analysis, Vol. 2, No. 4, 2005, pp. 232-234. doi:10.1287/deca.1050.0055

[19] L. A. Zadeh, “The Concept of a Linguistic Variable and Its Application to Approximate Reasoning," Information Science, Vol. 8, No. 3, 1975, pp. 199-249. doi:10.1016/0020-0255(75)90036-5

[20] H. M. Saraoglu and S. Sanli, “A Fuzzy Logic-Based Decision Support System on Anesthetic Depth Control for Helping Anesthetists in Surgeries,” Journal of Medical Systems, Vol. 31, No. 6, 2007, pp. 511-519.

[21] VDI 4707 Guideline, “Lifts Energy Efficiency,” 2008.
[22] G. Barney, "Vertical Transportation in Tall Buildings," Elevator World, Vol. LI, No. 5, 2003, pp. 66-75.

[23] CIBSE, "Guide D Transportation Systems in Buildings," 2005.

[24] J. Nipkow, "Electricity Consumption and Efficiency Potentials of Lifts,” Report of Swiss Agency for Efficient Energy Use SAFE, HTW Chur University of Applied Sciences, Zurich, 2005.

[25] N. Spyropoulos and L. Asvestopoulos, "Hydraulic vs. Traction Lifts: Environment Friendliness and Quality of Service to the User," The 17th International Congress on Vertical Transportation Technologies, Thessaloniki, 1113 June 2008, pp. 247-251.

[26] N. Mutoh, Y. Hayano, H. Yahagi and K. Takita, "Electric Braking Control Methods for Electric Vehicles with Independently Driven Front and Rear Wheels,” IEEE Transactions on Industrial Electronics, Vol. 54, No. 2, 2007, pp. 1168-1176. doi:10.1109/TIE.2007.892731

[27] M.-J. Yang, H.-L. Jhou, B.-Y. Ma and K.-K. Shyu, “A CostEffective Method of Electric Brake with Energy-Regeneration for Electric Vehicles,” IEEE Transactions on Industrial Electronics, Vol. 56, No. 6, 2009, pp. 2203-2212. doi:10.1109/TIE.2009.2015356 